\title{
A NOTE ON $L(p, q)$ SPACES AND ORLICZ SPACES WITH MIXED NORMS
}

\author{
MARIO MILMAN
}

\begin{abstract}
Necessary and sufficient conditions are given for the embedding of $L(p, q)$ spaces and Orlicz spaces with mixed norms.
\end{abstract}

1. Introduction. Let $X(0, \infty), Y(0, \infty), Z((0, \infty) \times(0, \infty))$ be Banach function spaces (cf. [6]) of Lebesgue measurable functions on $(0, \infty)$ (respectively $(0, \infty) \times$ $(0, \infty))$. Let $M$ denote the class of real valued Lebesgue measurable functions on $(0, \infty) \times(0, \infty)$ and let $[X, Y]=\left\{k \in M:\|k\|_{[X, Y]}<\infty\right\}$, where

$$
\|k\|_{[X, Y]}=\sup \left\{\iint|k(x, y) f(y) g(x)| d x d y:\|f\|_{X}<1,\|g\|_{Y^{\prime}} \leqslant 1\right\} .
$$

Therefore, if $k \in[X, Y]$ then $z_{k}(f)=\int k(x, y) f(y) d y$ defines a bounded linear operator $z_{k}: X \rightarrow Y$.

In the qualitative theory of integral equations (cf. [2]) it is important to determine necessary and sufficient conditions for a continuous embedding $Z \subseteq[X, Y]$ to hold. It is well known, and easy to see, that $Z$ is continuously embedded in $[X, Y]$ if and only if $X \otimes_{\pi} Y^{\prime}$ is continuously embedded in $Z^{\prime}$ (cf. [8]).

In [10] the following results are obtained for tensor products of $L(p, q)$ spaces and Orlicz spaces (the symbol $\subseteq$ will be used to denote a continuous embedding).

Theorem A. Let $A, B$ and $C$ be Young's functions, then the following statements are equivalent.

(i) $\exists \theta>0 \ni A^{-1}(t) B^{-1}(s) \leqslant \theta C^{-1}(t s), \forall t, s>0$,

(ii) $L_{A} \otimes_{\pi} L_{B} \subseteq L_{C}$,

(iii) $L_{A} \otimes_{\pi} M\left(L_{B}\right) \subseteq M\left(L_{C}\right)$.

TheOREM B. Let $1<p<\infty, 1 \leqslant q_{i} \leqslant \infty, i=1,2,3$. Then,

$$
L\left(p, q_{1}\right) \otimes_{\pi} L\left(p, q_{2}\right) \subseteq L\left(p, q_{3}\right)
$$

if and only if the following conditions are satisfied.

(i) $\max \left\{q_{1}, q_{2}\right\} \leqslant q_{3}$,

(ii) $1 / p+1 / q_{3} \leqslant 1 / q_{1}+1 / q_{2}$.

In this paper we consider $L(p, q)$ spaces and Orlicz spaces with mixed norms. Let $X(Y)$ denote the space defined as follows.

$$
X(Y)=\left\{f \in M:\|f\|_{X(Y)}=\|\| f(x, \cdot)\left\|_{Y}\right\|_{X}<\infty\right\} .
$$

Received by the editors March 14, 1980.

AMS (MOS) subject classifications (1970). Primary 46E30.

Key words and phrases. $L(p, q)$ spaces, Orlicz spaces, tensor products, mixed norms. 
It is clear that $X \otimes_{\pi} Y \subseteq X(Y)$ and therefore we have

$$
X(Y) \subseteq Z \Rightarrow X \otimes_{\pi} Y \subseteq Z .
$$

We prove the following

THeOREM 1. Let $A, B$ and $C$ be Young's functions, then the following statements are equivalent.

(i) $\exists \theta>0 \ni A^{-1}(t) B^{-1}(s) \leqslant \theta C^{-1}(t s), \forall t, s>0$,

(ii) $L_{A}\left(L_{B}\right) \subseteq L_{C}$,

(iii) $L_{A}\left(M\left(L_{B}\right)\right) \subseteq M\left(L_{C}\right)$.

Theorem 2. Let $1<p<\infty, 1 \leqslant q_{i} \leqslant \infty, i=1,2$, 3. Then, $L\left(p, q_{1}\right)\left(L\left(p, q_{2}\right)\right) \subseteq$ $L\left(p, q_{3}\right)$ if and only if the following conditions are satisfied.

(i) $\max \left\{q_{1}, q_{2}\right\} \leqslant q_{3}$,

(ii) $q_{1} \leqslant p \leqslant q_{3}$.

The sufficiency of the conditions of Theorem 2 was obtained by Walsh [11].

The reader will be assumed familiar with the theory of $L(p, q)$ spaces and Orlicz spaces (cf. [10], [5]). We shall follow the notation of [10]. Note, however, that the Marcinkiewicz $M_{A}$ spaces of [10] are denoted by $M\left(L_{A}\right)$ in the present work.

2. Proof of Theorem 1. We begin by recalling a construction of A. P. Calderón [1] which plays an important role in the theory of tensor products of function spaces (cf. [8]).

Let $(\Omega, \mu)$ be a measure space, $X(\Omega)$ a Banach function space, $\Sigma(X)$ its unit ball, and $A$ a Young's function. For each pair $(X(\Omega), A)$ define

$$
A^{-1}(X)(\Omega)=\left\{f \in M(\Omega): \exists \lambda>0 \text { and } g \in \Sigma(X) \ni|f(x)|<\lambda A^{-1}(|g(x)|)\right\}
$$

equipped with its natural norm $A^{-1}(X)$ becomes a Banach function space. Observe that $A^{-1}\left(L^{1}\right)=L_{A}$ and $A^{-1}(L(1, \infty)) \cong M\left(L_{A}\right)$ if $A$ satisfies the $\nabla_{2}$ condition.

The following result was obtained in [8].

(2.1) Lemma. Let $X, Y$ and $Z$ be Banach function spaces (as in §1), and $A, B$ and $C$ Young's functions. Moreover, assume that condition (i) of Theorem A is satisfied, then,
(i) $X \otimes_{\pi} Y \subseteq Z \Rightarrow A^{-1}(X) \otimes_{\pi} B^{-1}(Y) \subseteq C^{-1}(Z)$,
(ii) $X(Y) \subseteq Z \Rightarrow A^{-1}(X)\left(B^{-1}(Y)\right) \subseteq C^{-1}(Z)$.

Now observe that $L^{1}\left(L^{1}\right)=L^{1}$ and use (2.1) to obtain the equivalence of (i) and (ii). Moreover, since $L^{1}(L(1, \infty)) \subseteq L(1, \infty)$, (2.1) implies the equivalence of (i) and (iii), if we put the additional condition that $B$ and $C$ satisfy the $\nabla_{2}$ condition. To remove this condition we use the following.

(2.2) LeMMA (CF. [9]). Let $X, Y$ and $Z$ be rearrangement invariant spaces (as in §1). Suppose that there exists a constant $M>0$ such that $\forall u \in L^{1}(0, \infty)$ we have

$$
\left\|\phi_{Y^{\prime}}(|u|)\right\|_{X^{\prime}}<M \phi_{Z^{\prime}}\left(\|u\|_{1}\right)
$$

where $\phi_{Y^{\prime}}$ (respectively $\phi_{Z^{\prime}}$ ) denotes the fundamental function of $Y^{\prime}$ (respectively $Z^{\prime}$ ) (cf. [12]). Then,

$$
X(M(Y)) \subseteq M(Z) .
$$


In order to prove the implication (i) $\Rightarrow$ (iii) we apply (2.2). In fact a simple computation shows that (i) implies $\phi_{L_{\bar{\xi}}}(s) / \phi_{L_{\bar{c}}}(t) \leqslant \theta \bar{A}^{-1}(s / t), \forall t, s>0$. Therefore,

$$
\bar{A}\left(\phi_{L_{\bar{B}}}(s) / \theta \phi_{L_{\bar{c}}}(t)\right)<s / t, \quad \forall t, s>0 .
$$

Let $u \in L^{1}(0, \infty)$, then by the above inequality, we have

$$
A\left(\phi_{L_{\bar{B}}}(|u(x)|) / \theta \phi_{L_{\bar{c}}}\left(\|u\|_{1}\right)\right) \leqslant|u(x)| /\|u\|_{1} \quad \text { a.e., }
$$

which readily implies that $\left\|\phi_{L_{\bar{B}}}(|u|)\right\|_{L_{\bar{A}}} \leqslant \theta \phi_{L_{C}}\left(\|u\|_{1}\right)$. Therefore (iii) holds by (2.2).

The reverse implication (iii) $\Rightarrow$ (i) is trivial and follows from Theorem $A$ and (1.1).

(2.3) REMARK. For Orlicz spaces defined on finite measure spaces the equivalence (i) $\Leftrightarrow$ (ii) was proved in [4]. We point out that similar results hold for Orlicz-Marcinkiewicz spaces defined by generalized Young's functions (cf. [10], [8]).

3. Proof of Theorem 2. For the sake of completeness we prove that the conditions (i) and (ii) are sufficient. Let $p^{\prime}$ be defined by $1 / p+1 / p^{\prime}=1$, and let $u \in$ $L^{1}(0, \infty)$, then $\left\||u|^{1 / p^{\prime}}\right\|_{p^{\prime}}=\|u\|_{1}^{1 / p^{\prime}}$. Therefore, by $(2.2), L^{p}(L(p, \infty)) \subseteq L(p, \infty)$ (cf. [11]). Suppose now that (i) and (ii) hold, consider two cases: $q_{2} \leqslant p$ or $q_{2}>p$. In the first case we have

$$
L\left(p, q_{1}\right)\left(L\left(p, q_{2}\right)\right) \subseteq L^{p} \subseteq L\left(p, q_{3}\right) .
$$

In the second case we obtain the desired result interpolating (by the complex method) between $L^{p}(L(p, \infty)) \subseteq L(p, \infty)$ and $L^{p}\left(L^{p}\right)=L^{p}$ (cf. [5]).

The necessity of (i) follows from (1.1) and Theorem B, while the necessity of (ii) will follow from (3.1) and (3.2) below.

We use some constructions of Cwikel [3].

(3.1) LeMMA. $L\left(p, q_{1}\right)\left(L\left(p, q_{2}\right)\right) \subseteq L(p, \infty) \Rightarrow q_{1} \leqslant p$.

Proof. Consider two cases: $q_{2}=\infty$ or $q_{2}<\infty$. In the first case we get $q_{1}<p$ using (1.1) and Theorem B. Suppose now that $q_{2}<\infty$ and $q_{1}>p$. We shall construct $f \in L\left(p, q_{1}\right)\left(L\left(p, q_{2}\right)\right)$ such that $f \notin L(p, \infty)$.

Let us choose $0<\varepsilon<1$ such that $p<\varepsilon q_{1}$, and define (cf. [3])

$$
f(x, y)=\chi_{(0, e(x))}(y) F(x), \quad x, y \in(0, \infty),
$$

where $e(x)=(x+1)^{-1}, F(x)=\min \left\{1,[\log (x+1)]^{-\varepsilon / p}\right\}$. Then,

$$
\|\| f(x, \cdot)\left\|_{p, q_{2}}\right\|_{p, q_{1}}^{q_{1}} \sim \int_{e-1}^{\infty}(1+x)^{-q_{1} / p}[\log (x+1)]^{-\varepsilon q_{1} / p} x^{q_{1} / p} \frac{d x}{x}<\infty .
$$

On the other hand,

$$
\begin{aligned}
\lambda_{f}(t) & =|\{(x, y): 0<y \leqslant e(x), F(x)>t\}| \\
& =\log \left(\lambda_{F}(t)+1\right) .
\end{aligned}
$$

Therefore, $f^{*}(t)=F\left(e^{t}-1\right)$. Thus, for $t \geqslant 1, f^{*}(t)=t^{-\varepsilon / p}$ and

$$
\|f\|_{p, \infty}=\sup _{t>0}\left\{f^{*}(t) t^{1 / p}\right\}>\sup _{t>1}\left\{t^{-e / p} t^{1 / p}\right\}=\infty
$$


(3.2) LEMMA. If $L\left(p, q_{1}\right)\left(L\left(p, q_{2}\right)\right) \subseteq L\left(p, q_{3}\right)$, then $p<q_{3}$.

Proof. Assume without loss that $q_{3}<\infty$. Suppose that $L\left(p, q_{1}\right)\left(L\left(p, q_{2}\right)\right) \subseteq$ $L\left(p, q_{3}\right)$ but to the contrary $q_{3}<p$.

Let us choose $0<\varepsilon<1-q_{3} / p$, and define $f$ as in (3.1) with

$$
e(x)=e^{x}, \quad F(x)=e^{-x / p} \chi_{(0,1)}(x)+e^{-x / p} x^{-1 / p-e / q_{3}} \chi_{[1, \infty)}(x) .
$$

Then, as shown by Cwikel [3], $\|f\|_{p, q_{3}}=\infty$. However,

$$
\|\| f(x, \cdot)\left\|_{p, q_{2}}\right\|_{p, q_{1}}^{q_{1}} \sim \int_{1}^{\infty} x^{-\varepsilon q_{1} / q_{3}} \frac{d x}{x}<\infty
$$

a contradiction.

To complete the proof of Theorem 2 proceed as follows. Suppose $L\left(p, q_{1}\right)\left(L\left(p, q_{2}\right)\right) \subseteq L\left(p, q_{3}\right)$, then $L\left(p, q_{1}\right)\left(L\left(p, q_{2}\right)\right) \subseteq L(p, \infty)$. Now apply (3.1), (3.2), (1.1) and Theorem B to obtain the necessity of conditions (i)-(ii).

(3.3) REMARK. In our announcement [7], condition (ii) of Theorem 2 is incorrectly stated as $\max \left(q_{1}, q_{2}\right) \leqslant p \leqslant q_{3}$.

\section{REFERENCES}

1. A. P. Calderon, Intermediate spaces and interpolation, the complex method, Studia Math. 24 (1964), 113-190.

2. C. Corduneanu, Integral equations and stability of feedback systems, Academic Press, New York, 1973.

3. M. Cwikel, On $\left(L^{p^{Q}}\left(A_{0}\right), L^{P_{1}}\left(A_{1}\right)\right)_{\theta ; q}$, Proc. Amer. Math. Soc. 44 (1974), 286-292.

4. T. K. Donaldson, Inhomogeneous Orlicz-Sobolev spaces and nonlinear parabolic initial value problems, J. Differential Equations 16 (1974), 201-256.

5. R. A. Hunt, On $L(p, q)$ spaces, Enseign. Math. 12 (1966), 249-276.

6. W. A. J. Luxemburg, Banach function spaces, Thesis, Delft Inst. of Technology, Assen, 1955.

7. M. Milman, Embeddings of $L(p, q)$ spaces and Orlicz spaces with mixed norms, Notices Amer. Math. Soc. 24 (1977), A-533.

8. 147-149.

9. __ Embeddings of Lorentz-Marcinkiewicz spaces with mixed norms, Anal. Math. 4 (1978), 215-223.

10. R. O'Neil, Integral transforms and tensor products of Orlicz spaces and $L(p, q)$ spaces, J. Analyse Math. 21 (1968), 1-276.

11. T. Walsh, On $L^{p}$ estimates for integral operators, Trans. Amer. Math. Soc. 155 (1971), 195-215.

12. M. Zippin, Interpolation of operators of weak type between rearrangement invariant function spaces, J. Funct. Anal. 7 (1971), 267-284.

Department of Mathematics, University of Brasilia, 70.000 Brasilia, D. F., Brazil 